\section{Nuclear physics}

SEVENTEEN volumes are now available in the Oxford Physics Series (Oxford University Press: Oxford) edited by E. J. Burge D. J. E. Ingram and J. A. D. Matthew, and they already fulfil part of the declared aim of covering "in a flexible way, the material required for degree courses in pure physics, or physics combined with other subjects". The series as a whole is closely integrated, and Professor E. J. Burge's book, Atomic Nuclei and their Particles (hardback $£ 6$; paperback $£ 3.25$ ), together with that of G. K. T. Conn on Atoms and their Structure (forthcoming), provides a comprehensive and readable introduction to atomic and nuclear physics for first and beginning secondyear undergraduates. Together with D. J. E. Ingram's book on Radiation and Quantum Physics (hardback $£ 2.90$; paperback £1.45) they provide the basis for G. A. Jones' more advanced text on The Properties of Nuclei (hardback £5.95).

Burge's book begins with an elementary discussion of atoms, electrons and photons, and reviews the early history of the development of nuclear physics, with chapters on the nucleus revealed by alpha particles, and nuclear accelerators and instruments. The remainder of the book is devoted to nuclear reactions, nuclear forces and models, cosmic rays, and elementary particles. It is clearly and skilfully written with just the right amount of detail, and is enlivened by historical remarks that will help to bring the subject alive to the reader.

After mastering Burge, students will be well prepared to tackle the more advanced text of G. A. Jones, written mainly for second- and third-year undergraduates. It begins with a general survey of nuclear properties, and goes on to consider nuclear forces and nuclear models in some detail. Chapters follow on the spontaneous decay of nuclei, in particular alpha and beta decay and electromagnetic transitions, and on nuclear reactions, fission and fusion. There are useful appendices on cross-sections, pairing, exchange forces and spin matrices.

The difficulties of treating these subjects at this level are overcome very successfully by judicious use of simplified calculations, illuminated by penetrating comments revealing a deep understanding of the subject. Indeed there are few professional nuclear physicists who would not find it a rewarding experience to read this book.

Taken together, these two books complement each other admirably and integrate well with the other books in the series. They are well equipped with problems for students to test their understanding of the subject. They deserve a warm welcome and should be widely used in physics courses in universities and other centres of higher education.
A word should be added on the method of production. Previous books in the series have been type-set and printed and the results are fully up to the high standards of the Oxford University Press. The books of Burge and Jones, however, are produced by reproduction from typescript. Although this is apparently necessary for reasons of economy, it must be said with regret that the overall effect is much less pleasing to the eye, making the books less easy to read. The lines are not justified or always evenly spaced; bad

\section{Semiconductor devices}

The Physics of Semicondiktor Devices. By D. A. Fraser. Pp. 148. (Oxford University Press: Oxford, London and New York, 1977.) £5.95.

Semiconductor Devices are made to operate by the use of a very wide range of sophisticated electrical tricks. Therefore, the task of compressing a treatment of the physics of the effects so used into a slim volume requires great skill in selection; and to make it easy to read and entertaining is even more of a challenge. Future generations of students will, I believe, say that $\mathrm{Mr}$ Fraser has met all of these challenges remarkably well at a level which will suit second-year degree students in physics or engineering. The book will also help people like myself, who have learnt solid-state physics in disconnected segments and wish to bring these pieces together. The fact that the book is one of a teaching series (Oxford Physic's Series) and will relate well to other members of this series on the solid-state and electrical circuits, will help all classes of learner.

The subject-matter covered is about the same as that treated in earlier textbooks by Grove ( 370 pages) or Sze ( 800 pages) and can be divided up into about 60 pages on basic semiconductor physics and simple device theory and 60 on more advanced device theory and the discussion of modern technology and its implications. More attention is paid here than in the books mentioned above to teaching, in the sense of noting possible difficulties facing the student and taking pains to clarify these. The informal but highly informative style in which this is done is worth many pages of equations. Apart from a well-conceived figure giving an impression of the transport of an electron through a solid lattice, including scattering at centres and interaction with phonons, none of the diagrams were very original, which was a disappointment considering the authors expressed predilection for pictorial explanations. The art editor has also made things harder by using lettering which sometimes fades badly under the lithographic reproduction process used. breaks abound; there are fewer words per page; mathematical formulae are particularly clumsy; and the bubble chamber photograph on page 90 of Burge's book is illegible. The Oxford University Press deserves to be judged by the highest standards, and it is to be hoped that they will soon find ways of production that are both economical and worthy of their distinguished tradition. P. E. Hodgson

P. E. Hodgson is Lecturer in Nuclear Physics at the University of Oxford, $U K$.

The technical evaluation of such a brief summary must, of course, turn on what was left out as much as what was included. Let us start by saying that all of the derivations of semiconductor equations which were included were very skilfully stripped down to their essentials, with useful suggestions as to how the student can bridge any of the reasonably-sized jumps from point to point. I would have only a few quibbles, such as the use of $W$ for the potential energy of an electron and the showing of a complementary MOS circuit with an n-type well rather than the nearly universal p-type well.

The only omission which I felt to be considerable, even on the present scale, was the usual expression for commonemitter forward gain of a bipolar transistor in terms of emitter efficiency and of the two important forms of recombination -surface and depletion-region. The Ebers-Moll model of the transistor is presented very convincingly, followed by the hybrid- $\pi$ representation, in a form which does not distinguish surface and bulk recombination. This may have been chosen because the author chose to deal with real bulk recombination effects later, when dealing with thyristors. He also notes, in a later section on surfaces, that he will avoid discussion of real surface states. My personal opinion is that surface recombination is a bit too important to omit from any book dealing with technology of as well as the basics of semiconductor physics. What is more, the bipolar transistor, quite rightly, is treated as the core of the book and hence could be used nicely as the door to all of the technological problems discussed later on. This is not to say that the treatment given to the bipolar transistor is not, as far as it goes, illuminating.

This is a book which, because of its free-flowing, simplified treatment of some rather stiff physical principles, will help many students to overcome their difficulties with solid-state physics and give them the confidence to derive their own simple equations before forging on to the complexities of electronic technology.

Andrew Holmes-Siedle

Andiew Holmes-Siedle is a consultant to the Physics Department of Fulmer Research Institute and to the European Space Agency, on nuclear radiation techniques, solicl-state physics and space technology. 